\title{
Trust-based Routing Protocols in Wireless Networks
}

\author{
${ }^{1}$ S. SriGowthem, ${ }^{2}$ Dr. V. Khanna, ${ }^{3}$ Dr. K. P. Kaliyamurthie, \\ ${ }^{1}$ Research scholar, Department of CSE, Bharath University, Chennai. \\ ${ }^{2}$ Dean-Info, Bharath University, Chennai. \\ ${ }^{3}$ Professor \& Head, Department of CSE, Bharath University, Chennai.
}

Article History: Received: 11 January 2021; Accepted: 27 February 2021; Published online: 5 April 2021

ABSTRACT; the Wireless sensor Networks is usually consisting of all-inclusive amount of bound sensor
accessories which are announced in balance of the wireless media. As WSN's become added and added capital to
accustomed life, their aegis and assurance become a primary concern. However because of the attributes of WSNs,
aegis architecture can be challenging. Trust-aware acquisition protocols play a capital role in aegis of Wireless
Sensor Networks (WSNs). The key allotment of the acquisition agreement is avenue alternative based on account
theory. Our after-effects announce that trust-based geographic acquisition approaches the ideal achievement akin
accessible by flooding-based acquisition in bulletin supply arrangement and bulletin adjournment after incurring
abundant bulletin overhead.

Keywords: wireless sensor networks, multipath routing, Trust management, performance analysis, heterogeneous

\section{WSN (HWSN).}

The Confirmation course of action is a vanguard band-help for movement a base aegis related amidst two substances enduring endeavors or co operations aural a convey structure. Assertion association arrangements are assembled into three classes: united, impart and hybrid. Fused assertion association (CTM) schemes1 withstand of an individual all around trusted server that chooses the affirmation ethics of each irregularity in the framework. This gives the record of base computational ethereal at the sensor swell in light of the way that a lot of the attestation including is performed at concentrated trusted server that has no goals of computational capacity and memory. This passage about has the drawbacks of an individual motivation behind frustration, which makes it atomic strong. Furthermore, it smothers the basal reality that changed center points may recognize altered affirmation ethics around a correct regular center point.

\section{INTRODUCTION}

A Wireless Sensor Networks (WSNs) of spatially convey free sensors is changed in accordance with advice concrete or science rise, for instance, temperature, sound, weight, et cetera and to accommodatingly pig out their altered syntheses through the approach to a capital territory. The change of remote sensor frameworks was stirred by strong applications, for instance, bleeding edge observation; today such frameworks are usual in prospering modernized and customer applications, for instance, robotized movement condition and control, mechanical gathering bloom checking, and so on. The extra forefront frameworks are bi-directional, additionally enabling order of sensor activities.

The WSN is intrinsic of "center points" - from a couple to a couple of hundreds or even thousands, territory celebration bump is related to one (or once in a while a couple of) sensors. Celebration such sensor swell has around a couple areas: a radio handset with a joined getting wire or union to a pariah gathering device, a microcontroller, a computerized dealing with a record ambit for interfacing with the sensors and a development source, generally a show or a secured life structures of activity social occasion. Remote sensor frameworks are bound enduring affirmation as a result of the way that they are potentially low whole responses for an assortment of aggregate apple challenges. In any case, Sensor hubs acknowledge bound power, anamnesis and computational adroitness and suitably are available to catch. These systems are influenced to harsh solid elevation and assaults from enemies. So we charge the Circulated Assurance Management (DTM) schemes2 too don't orchestrate solid for complete sensor frameworks. In the impart approach, each knot locally figures the affirmation ethics of every one of extra centers over the span of activity that grows the computational cost. Also remembrance swell needs to impel a next to each other sequential registry about the certification ethics of the inside and out strategy in the life frameworks of a table. Advertisement estimation of the table is anon in respect to promotion estimation of the game-plan which deferred results in satisfactory anamnesis use. Celebration sensor swell keeps up its own specific affirmation ordered registry that gives the record of underneath direction airborne in light of the fact that knot does not blame to partner 
for some united server. Convey get to is incorporated strong than the united one since it has no individual motivation behind disillusionment.

Cream Assurance Administration (HTM) schemes3 oblige the setting of both thought as physically fit as convey affirmation association approaches. The capital cool of this get the chance to can't avoid being to decrease the entirety associated with certification examination when stood out from impart approaches. This game-plan is balanced with ingestion arranges, in which display bend goes about as a center point server for the refined pack. This get to is incorporated tried and true than the consolidated one yet underneath strong than the convey one. Each protuberance needs to push the sequential registry of alone accomplice centers, which gives the record of underneath anamnesis blasting than the convey approach. For intra-pack correspondence, center points charge to relate the show head. It presents included insight aeronautical throughout activity when diverged from the impart one.

The elocution of attestation association systems in WSN has been regular by the test assemble, and there endure multiplying approaches that run with the start of an anatomic and missing the mark structure. Regardless, thriving of these procedures don't yield into yearly some specific appearance of WSN that can access over their building and helpfulness. It is the explanation behind this cardboard to secure confident affirmation based acquirement traditions from the specific appearance of WSN, and to test the uninvolved assent of the through and through reinforcement of the workmanship on certification based getting with those endorsed systems. As an achievement of this examination, we can inspect those points of view that charge to be incorporated made in present and moving toward structures.

\section{RELATED WORK}

Ask about course of action on confirmation based securing plans for WSNs is in its start. To the extent anybody is concerned, bona fide few affirmation based getting plans recognize been proposed, for instance, RFSN4, ATRM5, PLUS6 and GTMS7.

Ganeriwal and Srivastava4 proposed RFSN, go recognition SN keeps up the value for adjoining center points so to speak. Trust ethics are impacted on the base of that value and they use Bayesian start for apery sufficiency of a center point. RFSN expect that the bump has ample associations with the neighbors so that the sufficiency (beta scattering) can limit a secured state. Regardless, if the measure of protuberance progress is higher, appropriateness direct won't settle. In RFSN, no

Protuberance is regular to advertise horrendous suitability information. If it is affected that "loathsome" value is around included by not giving out commendable reputation, again things being what they are, the blueprint won't have the ability to adjust to obscure conditions.

Boukercheeal.5 recognize proposed an ATRM course of action for WSNs. ATRM relies on upon an amassed WSN and learns confirmation in a totally impart way. ATRM tackles specific pro based stage. Similarly, it expect that there is an individual confided in power, which is manageable for repeating and washing adaptable administrators, which makes it accessible fringe an individual reason for failure.1 ATRM as well acknowledge that flexible authorities are vaporous add shocking centers that endeavor to grab or change guide instigated by the expert. In multiplying applications, this affirmation may not be commonsense.

Yao et al. 6 recognize proposed PLUS for sensor strategy security. The makers recognize a limited convey get to and assertion is impacted in light of either aggregate or atypical recognitions. This strategy tackles top of their own believable securing game-plan charged PLUS_R. In this arrangement, the makers recognize that all the fundamental specialist bundles created by the BS need to oblige a hashed game-plan total (HSN). Thought of HSN in power packages by all account not the only one extends the advertisement estimation of groups enduring in school bursting of manual and advancement limit however also manufactures the computational aggregate at the SNs. Also, at whatever point an adjudicator swell gets a package from choice swell i, it will dependably examination the authenticity of the bundle. If the openness examination flops, again the affirmation measure of bump i will be decreased free of whether irregularity $i$ was absolutely bewildering in harmfully authoritative some adjustment in a package or not. Thusly, swell i may get self-confident discipline.

Riaz Ahmed Shaikh proposed GTMS7, which uses gathering. This get to diminish the measure of affirmation evaluation. GTMS evaluates the affirmation of an accumulation of SNs in hostile to satisfactory certification association plans that dependably focus on affirmation ethics of alone center points. This get to gives us the record of serious underneath anamnesis to abundance affirmation file at celebration sensor swell in the framework.

\section{DEFINITION OF TRUST IN WSN}

Starting at now, the bigger piece of certification based systems balanced in remote correspondences mechanical get together computational affirmation models as demonstrated by the basal qualities of trust. Tragically, while an impressive measure of the affirmation dynamic running with to remote exchanges appearance suitability as a 
gathering wide astuteness of a center point's direct, the sobriquet "trust" is not dependably acclimated to ensure to the already specified thought. In8, affirmation is legitimate as the dread by an abettor swell about the aftereffects of the accomplishments of a capable irregularity in light of completed encounters and recognitions from attestant center points.

In9, assertion is true blue as an abettor center point's examination on how reasonable an attestant bump is in notice its affirmation decisions of the capable centers. While this basic is suggestive aural the vibe of the appearance in 9, it in all actuality clears the inclination force of the pondering of attestation and cloudy spots the projections amidst affirmation and validity which is an indispensable sharpness in the computational insistence composing. In spite of the basic of trust, a huge amount of insistence in impart affirmation and value mud of remote correspondences recognize that the steadfastness assessment of a mindful knot depends bountifully on the coterminous partner the abettor swell has with it. Exactly when there is a succinct release of such contiguous partner to consent for a suggestive dependability appraisal, buzz direct in the life frameworks of statements from attestant center points can be used. A recognized address for evaluating the loyalty of a capable bump B by an abettor protuberance An is to account apriori prescience of B tolerating an undertaking in an elegant deliver in the consecutive turn in light of the appeal available.

\section{TRUST INFORMATION COLLECTION}

In computational affirmation models in perspective of completed pivot experiences, the recorded accomplished practices of a subject, either add up to through inside and out recognitions or unusual examinations from others, life frameworks the declaration space.17 The capital issue of the accreditation ampleness is the portrayal of the master experiences. An attestation and sufficiency game-plan in the remote direction zone file remembrance turn possible result only. The compacted variant of industrious aggregator plenitude in an irregularity is generally referred to for the charge to address the turn history a crossed over. Along these lines, the multiplying totals of all by and large and revoking undertakings are changed in accordance with ordered registry completed evidences18. It is adjusted that the above example of the capable practices could be accessible in described a torpid change in the lead game-plan of a center point. In any case, with accomplished recognitions recorded as a gathered layout, this fragment of appeal is genuinely obscured. To for the most part give atonement for this, recognized affirmation models in remote correspondences generally ensure altered weights to the completed and the latest move comes about if after light the validation space.

\section{A. First Hand Information}

The test that action in the midst of the lifetime of a WSN can be usual as commitments for a certification association system, as they unique the direct of a confident sensor center point. A few difficulties are running with to the specific traditions that gadget the exact machine gave by the framework. 19Others are incorporated broadly comprehensive and endure late of the basal traditions and organizations: hardware related oversights, deviations from the sensor readings, issues in the guidance layer, inside and out imperativeness, et cetera. This impelled ambit of flanking direction should be taken into application if developing an attestation association structure: changed wellsprings of affirmation guidance will aftermath an extra solid affirmation system. 20

\section{B. Second Hand Information.}

Reputation direction about included center points should be dispersed, as this is a seriously fundamental grounds of affirmation association structures. Overlooking the usage of such buzz advice may deferred outcome in decisions that are not totally reliable with the incomparable reinforcement of the network.21 Besides, back a sensor knot is an article with bound knowledge, it can take in a deviation from its own direct and acclimate included centers about this exact introduction Nevertheless, in view of the open actuality of subverted center points irritating to downpour a horrendous face strike, an affirmation article for sensor frameworks must have the ability to suit genuine letters inspite of the truth of spurious and unpleasant reports.

\section{ANALYSIS OF EXISTING TRUST-BASED SYSTEM}

The place where there is certification association systems for WSN is palatable of absorption aural the examination relationship in the contempo years in spite of the way that it is still in a local state. An extensive measure of accomplishment has been done in the breadth of affirmation association structures for P2P or Ad Hoc networks10 for an examination on these systems. In any case, these structures don't redirect each one of the necessities and appearance appropriate by WSN. As stated, this examination broadness is attractive bona fide alive and a couple of diagrams recognize been produced.3 Still, prospering of the game plans are told with the reason concerning coherent genuine specific issues and a lot of them don't accord with all the appearance that an 
affirmation association strategy for WSN should give. In the going with, we will oblige a layout of the reinforcement of the workmanship in Assurance Administration for WSN. As a result of the convey characteristics of WSN, a lot of the incomparable assertion association systems refer to an impart affirmation unique which enables a subset of the center points to survey the lead of adjoining center points and accomplish decisions about them. The affirmation ethics are commonly picked up demography into application altered ambit, for instance, stated advertence (values got by coterminous move with the centers, too recognized as aggregate trust) and non-singular advertence (information secured from non-singular correspondence, too recognized as irregular trust). More present attestation association structures submit the truth of included parameters, for instance, dispositional affirmation (the principle some portion of accident the irregularity is accessible to take) 11 . As one of the capital errands of these systems is to model the direct of a center point, the truth of included ambit should be provoked as leeway.

The method for earth and gradual addition affirmation and adequacy is a swell genuine critical. The models can be real straightforward, expansiveness one can utilize disengaged morals, for example, 0 for un trusted or 1 for trusted or even one can utilize normal states, for example, normal trusted by application a sum in the midst of 0 and 1. Straightforward methodologies, for example, application a direct route activity are as well comprehensively progress in the expansiveness of affirmation for WSN. This is the situation of 11, broadness the confirmation sum is spoken to as an associated fanciful over a particular range, $(-1,+1)$. Application these direct route capacities, it is open to determine a start as per the mischance of the operation. Thusly, a decisive confirmation sum can be marked as untrusted or confided activatingly.

Concerning figuring of trust, some assertion association systems don't yield value into record, and anon get to the affirmation ethics as a multiplying aggregate of aggregate insistence and strange trust. 2 as the endorsement of an incident may be changed amidst celebratory centers the aplomb break is made about a prospering mean. The makers concur a framework for making the aplomb soften up perspective of the pivot and the including of adjusted weights. Included affirmation association systems 12 as well assent included outcast components that may impact insistence, for instance, how attestation well ordered incapacitates amidst additional items that no best collaborate. As value gives some holding settlements to confirmation association systems (e.g. more noteworthy association of perspectives, for instance, developing), some of them refer to reputation based frameworks zone centers move sufficiency for included centers and use it in acclimation to assess their steadfastness. For example, in4 sufficiency is addressed by a Bayesian definition, included especially, a beta suitability system. Another probabilistic action is acclimated in13 area the guide centers get the chance to advise about included center points by aggregate ascertainment and riches it as a beta association ambit tuple. Included systems use the Bayes supposition for social affair changed wellsprings of information. For instance, 14 introduces a Gaussian ampleness get ready for bond buzz direct from flanking center points with anon empiric information. Another office that recognize to be taken into yearly in any assertion association strategy is the region of the affirmation components, that is, the centers that can examination whether a definitive trustee can be trusted or not. In a lot of cases, any irregularity over the span of activity will have the ability to go about as an assertion article back each one of them recognize to abet show up a recognized target. The Base Station (BS) can too yield the piece of an insistence substance, as it has an all around appearance of the reinforcement of the system. All things considered, few confirmation organization frameworks agree the BS as an affirmation substance. One prime example is RDAT15, which proposes a model that assesses faithfulness of hubs in view of attributes, for example, detecting, obtaining and total. It makes utilization of a Beta worthiness framework, and every one of the figuring's and securing is done through the BS. Included get to that can be accustomed in change in accordance with convey the confirmation substances is to utilize bunches. Truth be told, some sensor systems aggregation their hubs into groups for arranged testimony (e.g. greater action organization or utilization of added capable hubs to kill meandering undertakings). The overseer of the exhibit is acknowledged as cluster head, and it can be in claim of moral story the affirmation premise and definitive mishap choices. This is the situation of TIBFIT16, an understanding that intends to discover and artificiality surmised swell disappointments in an occasion driven WSN zone the hubs are sorted out into groups. Still, not all bunch based frameworks residence affirmation article alone in the exhibit head. A model is7, range the creators consent a coming up short gathering based affirmation organization course of action that joins incorporated and communicate plans. Additionally, the exhibit curve is not in assertion of spreading exhortation about included hubs however the hubs themselves are in affirmation of securing counsel and scattering it a piece of their associate groups application a basic confirmation original in view of the worthiness of the hubs.

Another technique for scattering assertion substances is by in light of the way that pro based systems. ATRM5 is a master based insistence and value association game-plan domain the center points are not prepared to manage and handle their own particular affirmation and ampleness method. Thusly, the ATRM display requires that each protuberance locally holds an adaptable irregularity accountable for supervising affirmation and value on its encouraging center point. The approach relies on upon an amassed WSN with dauntlessness run its total is a flexible 
abettor structure. As ahead stated, some confirmation association systems are urged for analytic genuine specific issues. This is the circumstance of 14 , region the botheration of advancement has been dealt with by application groups whose bunch bend goes about as a hole amidst the display and the debased base (BS). Likewise, a couple attempts acknowledge been done in change in accordance with break the botheration of choosing the a ton of trusted cluster head $(8,7)$ application the basal confirmation organization course of action of the WSN. At last, some additional affirmation organization frameworks are record to be said as they familiarize new methodologies for overseeing confirmation and notoriety.

\section{CONCLUSION AND FUTURE WORK}

Remote Sensor Networks recognize been exact over again a genuine good blazon of frameworks. As the change and measure on this blazon of frameworks is up 'til now building up, the charge for including gadgets, for instance, affirmation or appropriateness is too creating. WSN will again pivot out to be incorporated good for incomparable apple applications. In this way, in this cardboard we recognize fabricated an analyze of balanced techniques for Assurance and Acceptability association systems for WSNs. Through this test we recognize specific a game plan of Best Practices that we submit as key and appropriate. We recognize these practices should be joined into the building of an attestation association game-plan for WSN. According to the distribution in light of these endorsed methodologies we recognize admonished which inside and out techniques for affirmation or suitability structures for WSN yield these practices into record.

The achievement of the affirmation association strategy limit depends on upon the affirmation of the practices. By good story the through and through systems we recognize appear to the altered pieces that some of these practices are by and large insulted by an extensive measure of the suggestion. This is the circumstance, for example, of affirmation and reputation. In a huge amount of the cases they are urged in like manner in acclimation to body the affirmation or ampleness systems. In the moving toward we plan to body missing the mark confirmation association structures for WSN that cover or, at atomic concur as multiplying of the acknowledged strategies said in this cardboard as could sensibly be normal.

\section{REFERENCES}

1. P. Resnick, R. Zeckhauser, E. Friedman, and K. Kuwabara. "Reputation systems: Facilitating trust in internet interactions". Comm. of the ACM, 43(12):pp.45-48, 2000.

2. Azzedine Boukerche, $\mathrm{Xu} \mathrm{Li}$, and K. EL-Khatib. "Trust-based security for wireless ad hoc and sensor networks". Computer Comm., 30:2413-2427, September 2007.

3. Sree Latha R., Vijayaraj R., Azhagiya Singam E.R., Chitra K., Subramanian V., "3D-QSAR and Docking Studies on the HEPT Derivatives of HIV-1 Reverse Transcriptase", Chemical Biology and Drug Design, ISSN : 1747-0285, 78(3) (2011) pp.418-426.

4. K. Krishna and A. bin Maarof." A hybrid trust management model for mas based trading society". The Int. Arab Journal of Information Technology, 1:60-68, July 2003.

5. S. Ganeriwal and M.B. Srivastava, "Reputation-Based Framework for High IntegritySensor Networks," Proc. ACM Workshop Security of Ad Hoc and Sensor Networks (SASN '04), pp. 66-67, Oct. 2004.

6. Masthan K.M.K., Aravindha Babu N., Dash K.C., Elumalai M., "Advanced diagnostic aids in oral cancer", Asian Pacific Journal of Cancer Prevention, ISSN: 1513-7368, 13(8) (2012) pp.3573-3576.

A. Boukerche, X. Li, and K. EL-Khatib, "Trust-Based Security for Wireless Ad Hoc and Sensor Networks," Computer Comm., vol. 30, pp. 2413-2427, Sept. 2007.

7. Z. Yao, D. Kim, and Y. Doh, "PLUS: Parameterized and Localized Trust Management Scheme for Sensor Networks Security," Proc.Third IEEE Int'l Conf. Mobile Ad-Hoc and Sensor Systems (MASS '06),pp. 437-446, Oct. 2006.

8. Tamilselvi N., Dhamotharan R., Krishnamoorthy P., Shivakumar, "Anatomical studies of Indigofera aspalathoides Vahl (Fabaceae)", Journal of Chemical and Pharmaceutical Research, ISSN : 0975 - 7384 , 3(2) (2011) pp.738-746.

9. R. A. Shaikh, H. Jameel, B. J. d'Auriol, H. Lee, S. Lee, and Y. J. Song, "Group-based trust management scheme for clustered wireless sensor networks," IEEE Transactions on Parallel and Distributed Systems, vol. 20, no. 11, pp. 1698-1712, 2009.

A. Boukerch, L. Xu, and K. EL-Khatib," BTrust-based security for wireless ad hoc and sensor networks", Comput. Commun., vol. 30, pp. 2411-2427, 2007.

10. Devi M., Jeyanthi Rebecca L., Sumathy S., "Bactericidal activity of the lactic acid bacteria Lactobacillus delbreukii", Journal of Chemical and Pharmaceutical Research, ISSN : 0975 - 7384 , 5(2) (2013) pp.176180 . 
11. S. Buchegger and J.-Y. L. Boudec, "BA robust reputation system for P2P and mobile ad-hoc networks", in Proc. 2nd Workshop Econom.Peer-to-Peer Syst., Cambridge, MA, 2004.

12. R. Roman, M. C. Fernandez-Gago, J. Lopez, and H.-H. Chen. "On Secu rity and Privacy in Mobile and Wireless Networking, chapter Trust and Reputation Systems for WSN". Troubador Publishing Ltd, 2009.

13. G. P. Navarrete M. Momani, J. Agbinya and M. Akache. "A New Algorithm of Trust Formation in Wireless Sensor Networks". In 1st IEEEInternational Conference on Wireless Broadband and Ultra Wideband Communciations (AusWireless'06), Sydney, Australia, March 2006.

14. C. Deokjai H. Guangjie and L. Wontaek. "A Reliable Approach of Establishing Trust for Wireless Sensor Networks". In IFIP International Conference on Network and Parallel Computing Workshops (NPC 2007),Dalian, China, September 2007.

15. F. Li A. Srinivasan and J. Wu. "A Novel CDS-Based Reputation Monitoring System for Wireless Sensor Networks". In 28th International Conference on Distributed Computing Systems Workshops (ICDCS 2008),Beijing, China, June 2008.

16. Reddy Seshadri V., Suchitra M.M., Reddy Y.M., Reddy Prabhakar E., "Beneficial and detrimental actions of free radicals: A review", Journal of Global Pharma Technology, ISSN : 0975-8542, 2(5) (2010) pp.3-11.

17. M. Momani, K. Aboura, and S. Challa. "RBATMWSN: Recursive Bayesian Approach to Trust Management in Wireless Sensor Networks". In 3rd International Conference on Intelligent Sensors, Sensor Networks and Information (ISSNIP 2007), pages 347-352, Dec. 2007.

18. S. Ozdemir." Functional Reputation based Reliable Data Aggregation and Transmission for Wireless Sensor Networks". Comput. Commun., 31(17):3941-3953, 2008.

19. M. Krasniewski and B. Rabeler. "TIBFIT: Trust Index Based Fault Tol- erance for Arbitrary Data Faults in Sensor Networks". In DSN '05: Proceedings of the 2005 International Conference on Dependable Systems and Networks, pages 672-681, Washington, DC, USA, 2005

20. Kimio T, Natarajan G, Hideki A, Taichi K, Nanao K. Higher involvement of subtelomere regions for chromosome rearrangements in leukemia and lymphoma and in irradiated leukemic cell line. Indian Journal of Science and Technology. 2012 April, 5 (1), 1801-1811.

21. Cunningham CH. A laboratory guide in virology. $6^{\text {th }}$ edn. Burgess Publication Company: Minnesota, 1973.

22. Sathishkumar E, Varatharajan M. Microbiology of Indian desert. In: Ecology and vegetation of Indian desert. D.N.Sen (ed.), Agro Botanical Publ.: India. 1990, 83-105.

23. Varatharajan M, Rao BS, Anjaria KB, Unny VKP, Thyagarajan S. Radiotoxicity of sulfur-35. Proceedings of 10th NSRP, India, 1993, 257-258.

24. Article title. http://www.indjst.org/index.php/vision. Date accessed: 01/01/2015. 